\title{
Die litauische Ratspräsidentschaft 2013: klein und effektiv
}

\author{
Ramūnas Vilpišauskas und Bruno Vandecasteele*
}

Litauen hatte seine erste rotierende Präsidentschaft des Rates der Europäischen Union vom 1. Juli bis 31. Dezember 2013 inne. Die Präsidentschaft begann unter schwierigen Umständen: Es war die letzte Legislaturperiode in voller Länge vor der Europawahl im Jahr 2014, die ausnahmsweise auch mit der letzten Ratspräsidentschaft für die Annahme der erforderlichen Rechtsakte zur Umsetzung des mehrjährigen Finanzrahmens 2014-2020 (MFR) zusammenfiel. Darüber hinaus wird die erste Präsidentschaft oft als ultimativer Test betrachtet, ob ein Land ein vollwertiges EU-Mitglied ist. Das relativ kleine Budget für die Präsidentschaft in Höhe von 62 Millionen Euro und die Tatsache, dass die neue Koalitionsregierung erst ein halbes Jahr vor dem Beginn der Präsidentschaft ins Amt kam, senkten die Erwartungen an die Effektivität der litauischen Präsidentschaft. Innerhalb dieser Rahmenbedingungen entwarf Litauen ein ehrgeiziges Arbeitsprogramm, mit dem Ziel eines ,glaubwürdigen, offenen und wachsenden Europas". 1

Der vorliegende Beitrag beurteilt die litauische Präsidentschaft in drei Schritten: Zunächst wird ein kurzer Überblick über die Vorbereitungen und die Festlegung der Prioritäten gegeben, wodurch aufgezeigt wird, wie Litauen zu EU-Themen steht und wie seine Regierung sich darauf vorbereitete, ihren Beitrag für die Europäische Union zu leisten. Zweitens werden die wichtigsten Erfolge der Präsidentschaft kurz vorgestellt, dabei wird besonderes Augenmerk auf die Ziele der Stärkung der Glaubwürdigkeit, der Offenheit und des Wachstums in der Europäischen Union gelegt. Der Beitrag konzentriert sich auf die wichtigsten Leistungen zur Erreichung jedes dieser Ziele. Dies sind die Schaffung einer europäischen Bankenunion, die Einigung über den neuen MFR und eine Stärkung der Beziehungen der Europäischen Union mit den Ländern der Östlichen Partnerschaft. Anschließend werden diese Erfolge mit den Rollen verbunden, die Litauen während seiner Präsidentschaft und in den verschiedenen von ihm bearbeiteten Dossiers spielte. Der Beitrag schließt mit einer Zusammenfassung der wichtigsten Ergebnisse sowie einigen Überlegungen zu den Auswirkungen der Präsidentschaft auf die Position Litauens in der Europäischen Union.

\section{Prioritäten und Vorbereitung auf die Präsidentschaft}

\section{Der nationale institutionelle und politische Rahmen}

Seit der letzten Reform des Systems zur Koordinierung der litauischen Europapolitik im Jahr 2009 nimmt das Ministerium für Auswärtige Angelegenheiten die Rolle eines Koordinators wahr. Es spielte auch während der Vorbereitung und Durchführung der Präsident-

* Prof. Dr. Ramūnas Vilpišauskas, Vilnius University, Direktor des Institute of International Relations and Political Science, Vilnius.

Bruno Vandecasteele, Doktorand, Centre for EU Studies, Ghent University, Stipendiat des Flemish Research Council (FWO).

1 Litauische Ratspräsidentschaft: Programme of the Lithuanian Presidency of the Council of the European Union 1 July to 31 December 2013: For a Credible, Growing and Open Europe, abrufbar unter: http://static.eu2013.1t/ uploads/documents/Programos/Programa_EN.pdf (letzter Zugriff: 1.7.2013). 
schaft eine zentrale Rolle, sowohl im Hinblick auf die Inhalte als auch auf die Logistik. ${ }^{2}$ Inhaltlich trug vor allem die Regierungskommission für EU-Angelegenheiten (Vyriausybès Europos Sajungos komisija, VESK) zur Festlegung der Prioritäten für die Ratspräsidentschaft bei. Bei der Regierungskommission für EU-Angelegenheiten handelt es sich um ein wöchentliches Treffen von allen stellvertretenden Ministern sowie von Mitarbeitern des Büros des Ministerpräsidenten und des EU-Botschafters, bei dem der Außenminister den Vorsitz führt. Die Sitzungen werden einerseits von der Abteilung für EU-Angelegenheiten des Ministeriums für Auswärtige Angelegenheiten und andererseits von einem KoordinatorenNetzwerk vorbereitet, das sich aus Vertretern der Seimas (dem litauischen Parlament), dem Präsidenten, dem Premierminister und den Ministerien zusammensetzt. Das KoordinatorenNetzwerk wird vom stellvertretenden Außenminister geleitet. Anlässlich der Präsidentschaft, richtete die litauische Regierung eine temporäre Abteilung für die Ratspräsidentschaft (Pirmininkavimo ES Tarybai Departamentas, PESTD) innerhalb des Ministeriums für Auswärtige Angelegenheiten ein. ${ }^{3}$ Die PESTD war für die Organisation, Planung, Kommunikation und Logistik aller Aktivitäten der Präsidentschaft verantwortlich. Es gab somit klare Verfahren für die Koordinierung zwischen den verschiedenen Ministerien, sowohl in Bezug auf inhaltliche Fragen als auch die Logistik. Die Befragten gaben in den Interviews an, dass diese Arbeitsteilung sehr produktiv war: Jede Abteilung konnte sich auf den von ihr verantworteten Teil der Arbeit konzentrieren. ${ }^{4}$

Die Vorbereitungen für die Präsidentschaft begannen drei Jahre im Voraus, zunächst durch Fortbildung des Personals und die Schaffung der institutionellen Strukturen. Im Oktober 2011 wurde ein wichtiger Schritt gemacht, als alle in der Seimas vertretenen Parteien sich verpflichteten, die Präsidentschaft nicht zum Gegenstand des Wahlkampfes im Vorfeld der Parlamentswahlen im Jahr 2012 zu machen, ${ }^{5}$ damit die Kontinuität der Vorbereitungen unabhängig von der Zusammensetzung der künftigen Regierung gewährleistet werden konnte. Die Wahlen führten zu einer Mitte-links-Koalition, geführt von den Sozialdemokraten, die eine von den Konservativen geführte Mitte-rechts Koalition ablöste. Die politischen Parteien hielten sich an ihre Vereinbarung aus dem Jahre 2011 und der Vorbereitungsprozess zeigte eine bemerkenswerte Kontinuität.

Während der eigentlichen Präsidentschaft kam dem Ministerium für Auswärtige Angelegenheiten weiterhin die Schlüsselrolle bei der Koordinierung aller Aktivitäten zu, aber auch die Ständige Vertretung Litauens bei der Europäischen Union in Brüssel leistete einen entscheidend Beitrag. Ihr Personal wurde auf bis zu 180 Beamte mehr als verdoppelt, was ein Viertel des Haushalts für die Ratspräsidentschaft absorbierte. ${ }^{6}$ In Fragen, die die europäischen Rechtsetzungsverfahren betreffen, wurde der Ständigen Vertretung viel mehr Auto-

2 Ramūnas Vilpišauskas/Bruno Vandecasteele/Austė Vaznonyte: The Lithuanian Presidency of the Council of the European Union. Advancing energy policy and Eastern Partnership goals: conditions for exerting influence, in: Lithuanian Foreign Policy Review 1/2013, S. 11-37.

3 Vadim Lucenko: Préparation de la présidence du Conseil: le cas de la Lituanie (second semestre 2013), in: Véronique Charléty/Michel Mangenot (Hrsg.): Le système présidentiel de l'Union européenne après Lisbonne, Strasburg 2011, S. 75-77.

4 Interview im litauischen Außenministerium, Vilnius, 24. Februar 2014; Interview im litauischen Außenministerium, Vilnius, 3. März 2014.

5 Seimas: Political Parties Represented at the Seimas Signed the Accord on Lithuania's EU Presidency in JulyDecember 2013, abrufbar unter: http://www3.lrs.lt/pls/inter/w5_show?p_r=8296\&p_d=116591\&p_k=2 (letzter Zugriff: 23.10.2013).

6 Litauische Ratspräsidentschaft: Lietuvos pasirengimas pirmininkavimui Europos Sajungos Tarybai, abrufbar unter: http://ec.europa.eu/lietuva/documents/power_pointai/metine2010/2012/pirmininkavimas_est.ppt (letzter Zugriff: 20.11.2013). 
nomie als gewöhnlich zugestanden. Litauens Beitrag zur EU-Außenpolitik, vor allem zur Östlichen Partnerschaft, wurde dagegen von Beamten in Vilnius geleitet. ${ }^{7}$ Während der Präsidentschaft zeigten sowohl die litauische Präsidentin Dalia Grybauskaitè als auch der litauische Außenminister Linas Linkevičius in diesen Fragen Führungsstärke.

\section{Ehrgeizige Prioritäten: Glaubwürdigkeit, Offenheit und Wachstum in der EU}

Im November 2011 äußerte sich der Seimas das erste Mal zu den künftigen Prioritäten der Präsidentschaft. ${ }^{8}$ Das Parlament schrieb fest, die litauische Regierung solle sich für dauerhaftes Wirtschaftswachstum, Energiesicherheit und die Verbesserung der europäischen Wettbewerbsfähigkeit durch die Schaffung von Arbeitsplätzen und weitere Haushaltskonsolidierung einsetzen. Darüber hinaus forderte es, dass die litauische Regierung sich während der Ratspräsidentschaft auf vier Schwerpunktbereiche konzentrieren solle: Erhöhung der Energiesicherheit in der Europäischen Union, die Wiederbelebung der Ostseestrategie, die Vertiefung der Beziehungen der Europäischen Union mit den Ländern der Östlichen Partnerschaft und die Gewährleistung einer wirksamen Kontrolle der Außengrenzen der Europäischen Union. Diese vier Schwerpunkte, insbesondere die Vertiefung der Beziehungen zu den östlichen Nachbarn und die Frage der Energiesicherheit, schließen an die europapolitischen Prioritäten Litauens an, die 2004 im Zuge des Beitritts des Landes zur Europäischen Union formuliert wurden. Es sollte auch darauf hingewiesen werden, dass viele Mitglieder der Seimas die Präsidentschaft zunächst als Chance sahen, um nationale Interessen zu verfolgen. Diese Wahrnehmung wandelte sich, als das Präsidentschaftsprogramm formuliert und die Abstimmung mit der irischen Ratspräsidentschaft Anfang 2013 intensiviert wurde. Zwar gab es auch ein Element der einfachen ,Neuverpackung' der Prioritäten der Präsidentschaft, aber es scheint, dass zumindest einige Politiker einen Lernprozess durchliefen und erkannten, dass die EU-Ratspräsidentschaft vor allem der Förderung europäischer Gesetzgebungsprozesse dient. Infolgedessen wurden die nationalen Interessen in drei große Ziele umformuliert: ${ }^{9}$ 1) ein ,glaubwürdiges Europa'; 2) ein ,wachsendes Europa' und; 3) ein ,offenes Europa'.

Unter dem Titel ,ein glaubwürdiges Europa‘, plante die Präsidentschaft mehrere Schritte zur Stabilisierung des Finanzsektors und zur Haushaltskonsolidierung sowie Initiativen in Richtung einer wachstumsorientierten Wirtschaftspolitik mit einer starken sozialen Dimension. Diese beinhalteten eine strikte Umsetzung der Vereinbarungen über soziale, wirtschaftliche und fiskalische Reformen, Bemühungen zur Schaffung einer Bankenunion durch Einrichtung eines einheitlichen Mechanismuses zur Bankenabwicklung und die Entwicklung von Instrumenten zur Bekämpfung von Steuerhinterziehung und Steuerbetrug.

Für ein ,wachsendes Europa ' wollte Litauen Investitionen in innovative Projekte fördern, den Binnenmarkt stärken, bessere Beschäftigungsmöglichkeiten schaffen und nachhaltige soziale Sicherungssysteme entwickeln. Das wichtigste dieser Ziele war es, den Gesetzgebungsprozess des neuen MFR abzuschließen, der rund 70 Rechtsakte beinhaltet. Die Präsidentschaft plante auch zur Schaffung eines gemeinsamen Raums des Wissens, der Forschung und Innovation beizutragen. Dazu sollte sichergestellt werden, dass die Umsetzung des Rah-

7 Interview in der Ständigen Vertretung Litauens bei der Europäischen Union, Brüssel, 19. Juni 2013.

8 Seimas: Seimo Nutarimo Dèl Lietuvos Respublikos pirmininkavimo Europos Sajungos Tarybai 2013 m. Projektas (XIP-3550), abrufbar unter: http://www3.lrs.lt/pls/inter2/dokpaieska.showdoc_1?p_id=409464\&p_query=\&p_tr 2= (letzter Zugriff: 30.3.2013).

9 Litauische Ratspräsidentschaft: Programme of the Lithuanian Presidency of the Council, 2013. 
menprogramms für Forschung und Innovation „Horizont 2020“ bereits 2014 beginnt und dass die digitale Agenda der Europäischen Union weiter gefördert wird. Außerdem plante Litauen mehrere Schritte zur Stärkung des Binnenmarkts: diese umfassten Garantien der Rechte entsandter Arbeitnehmer, eine Einigung über die Verordnung über elektronische Identifizierung und Vertrauensdienste für elektronische Transaktionen im Binnenmarkt, ${ }^{10}$ eine Vereinbarung über die Fazilität „Connecting Europe“"11 und die Vollendung des Energiebinnenmarkts. Außerdem wollte die litauische Regierung Maßnahmen zur Senkung der Jugendarbeitslosigkeit entwickeln und makroregionale Zusammenarbeit stärken.

Die dritte Priorität ,ein offenes Europa' zielte auf die Entwicklung einer Europäischen Union, die in der Lage ist, die globalen Herausforderungen zu bewältigen, demokratische Werte zu fördern, einen Beitrag für eine sichere Nachbarschaft zu leisten und die Rechte der EU-Bürger zu schützen. Die litauische Regierung erklärte, dass sie den Erweiterungsprozess fortsetzen, Freihandelsabkommen mit Drittländern fördern, Initiativen für einen besseren Schutz der EU-Außengrenzen ergreifen, Schmuggel bekämpfen und an einem Rahmen für globale Entwicklung für die Zeit nach 2015 mitarbeiten möchte.

Obwohl Litauen sich traditionell für die Erweiterung der Europäischen Union einsetzt, war die Östliche Partnerschaft der am besten ausgearbeitete außenpolitische Teil des Präsidentschaftsprogramms. Dieser umfasste drei Hauptkategorien: erstens, Litauen plante die Fortentwicklung des weiteren politischen Rahmens der EU-Beziehungen zur östlichen Nachbarschaft, einschließlich der Unterzeichnung eines Assoziierungsabkommens mit der Ukraine, der Paraphierung entsprechender Abkommen mit der Republik Moldau, Georgien und Armenien sowie die Fortentwicklung der Assoziierungsagenda mit Aserbaidschan. Zweitens, plante die litauische Regierung, den Kooperationsprozess der Östlichen Partnerschaft durch eine Ausweitung ihres Anwendungsbereichs auf weitere Politikfelder und durch die Einrichtung von High-Level-Dialogen in verschiedenen Bereichen aufzuwerten, um einen unumkehrbaren Prozess der ständigen Zusammenarbeit auf höchster Ebene zu beginnen. ${ }^{12}$ Drittens, wollte sie Reflexionen über die weiteren Entwicklungsmöglichkeiten der Östlichen Partnerschaft anregen; bis 2013 erschien die Unterzeichnung von Assoziierungsabkommen als höchste Stufe des Annäherungsprozesses zwischen der Europäischen Union und den Ländern der Östlichen Partnerschaft. Sobald diese Abkommen paraphiert oder unterzeichnet wurden, sollte eine Debatte darüber beginnen, was den Abkommen folgt und wie viel die Europäische Union - finanziell und politisch - bereit ist zu leisten, um diese Abkommen umzusetzen. ${ }^{13}$

\section{Die Erfolge der Präsidentschaft}

Die rotierenden Präsidentschaften messen ihre Leistungen üblicherweise anhand der Anzahl von Fällen, in denen eine Einigung über Dossiers durch die Präsidentschaft erreicht

10 Europäische Kommission: Vorschlag für eine Verordnung des Europäischen Parlaments und des Rates über die elektronische Identifizierung und Vertrauensdienste für elektronische Transaktionen im Binnenmarkt, KOM (2012) 238

11 Verordnung (EU) Nr. 1316/2013 des Europäischen Parlaments und des Rates vom 11. Dezember 2013 zur Schaffung der Fazilität „Connecting Europe“, zur Änderung der Verordnung (EU) Nr. 913/2010 und zur Aufhebung der Verordnungen (EG) Nr. 680/2007 und (EG) Nr. 67/2010, in: Amtsblatt der EU, Nr. L 348 vom 20. Dezember 2013, S. 129-171.

12 Interview im litauischen Außenministerium, Vilnius, 13. März 2013.

13 Interview im litauischen Außenministerium, Vilnius, 5. April 2013; Interview in der Ständigen Vertretung Litauens bei der Europäischen Union, Brüssel, 19. Juni 2013. 
wurde. Der litauische Vorsitz war in dieser Hinsicht sehr effektiv, nicht nur im Vergleich zu den vorangegangenen sowie den Präsidentschaften anderer Mitgliedstaaten aus der Erweiterungsrunde von 2004, sondern auch im Vergleich zu den Präsidentschaften, die während der gleichen Phase des Europäischen Wahlzykluses in den Jahren 2004 und 2009 amtierten. Nach Angaben der litauischen Beamten wurden 141 Rechtsakte und 283 Rechtsakte ohne Gesetzescharakter während des Vorsitzes beschlossen, mehr als das Doppelte des Durchschnitts der vorangegangenen Präsidentschaften. ${ }^{14}$ Es wurde auch betont, dass die Anzahl der Beschlüsse, die im ordentlichen Gesetzgebungsverfahren unter Beteiligung des Europäischen Parlaments gefasst wurden, mit $92^{15}$ die Zahl derer überstieg, die vor den letzten Wahlen zum Europäischen Parlament in den Jahren 2004 und 2009 beschlossen wurden. ${ }^{16}$

\section{Die Bankenunion: ein glaubwürdigeres Europa?}

Die litauische Präsidentschaft musste sich zwangsläufig mit der Krisenpolitik der Europäischen Union zur Bewältigung der Folgen der Wirtschaftskrise befassen, von denen die meisten Mitglieder der Eurozone bereits Jahre vor dem Beginn der litauischen Präsidentschaft betroffen waren. Wie der Europäische Rat vom 27. bis 28. Juni 2013 erklärte, hatte die Vollendung der Bankenunion, die von den EU-Institutionen im Jahr 2012 vorgeschlagen worden war, kurzfristig die höchste Priorität im Rahmen der Reform der Wirtschafts- und Währungsunion. ${ }^{17}$ Das Ziel der Bankenunion ist die Verabschiedung von einheitlichen Regelungen der Eigenkapitalvorschriften für Banken Überwachung, Restrukturierung und Auflösung von Banken in der Europäischen Union mit der Absicht, die Kosten für die Steuerzahler in den Mitgliedstaaten zu minimieren und den Teufelskreis aus Bankenrettung und Staatsschuldenkrisen zu durchbrechen. Der Einheitliche Bankenaufsichtsmechanismus, der der Europäischen Zentralbank (EZB) die Befugnisse verleiht, Banken in der Eurozone und anderen Mitgliedstaaten, die der Bankenunion beitreten wollen, direkt zu überwachen, wurde vor der litauischen Präsidentschaft ausgehandelt und trat im November 2013 in Kraft. Die größte Herausforderung, vor die sich die litauische Präsidentschaft gestellt sah, war es, eine Einigung über das andere wichtige Element der Bankenunion zu erreichen - den einheitlichen Mechanismus zur Bankenabwicklung. Diese sollte noch vor Ende des Jahres 2013 erreicht werden, damit die entsprechende Verordnung noch vor dem Ende der laufenden Legislaturperiode des Europäischen Parlaments im Jahr 2014 verabschiedet werden kann.

Im Juli 2013 präsentierte die Europäische Kommission die Verordnung über den einheitlichen Mechanismus zur Bankenabwicklung. ${ }^{18}$ Sie schlug vor, der EZB und der Europäischen Kommission im Rahmen eines Ausschusses für die einheitliche Abwicklung (dem auch nationale Behörden des Landes angehören, in dem die Bank ihren Sitz hat) das Initiativrecht zu übertragen ebenso wie Entscheidungsbefugnisse über die Abwicklung einer Bank

14 Dalia Grybauskaitė: Speech by President Dalia Grybauskaite to the European Parliament, 15.1.2014, abrufbar unter: http://www.eu2013.1t/en/news/statements/speech-by-president-dalia-grybauskaite-to-the-european-parliament (letzter Zugriff: 6.5.2014).

1587 Beschlüsse in erster Lesung und fünf in zweiter Lesung.

16 Lecture of the Ambassador Raimundas Karoblis, 25.3.2014, Institute of International Relations and Political Science, Vilnius University.

17 Europäischer Rat: Europäischer Rat (Tagung vom 27./28. Juni 2013). Schlussfolgerungen, EUCO 104/2/13.

18 Europäische Kommission: Vorschlag für eine Verordnung des Europäischen Parlaments und des Rates zur Festlegung einheitlicher Vorschriften und eines einheitlichen Verfahrens für die Abwicklung von Kreditinstituten und bestimmten Wertpapierfirmen im Rahmen eines einheitlichen Abwicklungsmechanismus und eines einheitlichen Bankenabwicklungsfonds sowie zur Änderung der Verordnung (EU) Nr. 1093/2010 des Europäischen Parlaments und des Rates, KOM (2013) 520. 
in ernsten finanziellen Schwierigkeiten. Der Vorschlag sah die Schaffung eines einheitlichen Abwicklungsfonds durch Beiträge aus dem Bankensektor vor, um die nationalen Rettungsfonds zu ersetzen, diskutierte aber auch die Möglichkeit der direkten Rekapitalisierung von Banken durch den Europäischen Stabilitätsmechanismus (ESM).

Der Vorschlag der Europäischen Kommission stimmte nicht mit den Interessen einiger EU-Mitgliedstaaten, insbesondere Deutschlands, überein. Die Hauptstreitpunkte waren die Rechtsform, die Reichweite der Verordnung, der ,financial backstop“ (oder eine zentralisierte Finanzierung), die Entscheidungsverfahren und die Befugnisse der Europäischen Union gegenüber den nationalen Institutionen. Obwohl der Verordnungsentwurf auf der Grundlage des Artikels 114 des Vertrags über die Arbeitsweise der Europäischen Union präsentiert wurde, was für die Annahme eine qualifizierte Mehrheit im Rat und die Zustimmung des Parlaments erforderlich macht, arbeitete die litauische Präsidentschaft an einem auch für Deutschland akzeptablen Kompromiss. Die Verhandlungen über den einheitlichen Mechanismus zur Bankenabwicklung drehten sich meist darum, die Vorschläge der Europäischen Kommission den Interessen Deutschlands anzunähern, wodurch dessen Bedeutung für den Abschluss der Bankenunion anerkannt wurde. ${ }^{19}$

Im Dezember 2013 einigte sich der Rat auf ein weiteres Vorgehen, welches sowohl den Entwurf der Verordnung zur Festlegung einheitlicher Vorschriften und eines einheitlichen Verfahrens für die Abwicklung von Kreditinstituten als auch eine Verpflichtung umfasste, bis zum 1. März 2014 eine zwischenstaatliche Vereinbarung über den Abwicklungsfonds zu schließen. Letztere sollte Bestimmungen über die nationalen Beiträge zum Abwicklungsfonds und die schrittweise zunehmende Deckung des Fondskapitals durch die auf nationaler Ebene erhobene Bankenabgabe beinhalten. ${ }^{20}$ Der Entwurf der Richtlinie zur Festlegung eines Rahmens für die Sanierung und Abwicklung von Kreditinstituten und Wertpapierfirmen ${ }^{21}$ sieht eine ,Bail-in'-Regel vor, also die Beteiligung von Anteilseignern an der Rettung einer Bank. Diese Richtlinie und die Richtlinie über Einlagensicherungssysteme ${ }^{22}$ wurden bereits im Dezember 2013 unter litauischer Präsidentschaft verabschiedet, welche zuvor auch die Einigung mit dem Europäischen Parlament aushandelte. Der einheitliche Mechanismus zur Bankenabwicklung umfasst alle Banken in den teilnehmenden Mitgliedstaaten, wobei der Ausschuss verantwortlich für die Auflösung der direkt von der EZB überwachten Banken und der grenzüberschreitenden Banken (130 Banken insgesamt) ist. Die nationalen Behörden sind für die Abwicklung aller anderen Banken verantwortlich (rund 6.200 Banken). Bei der Präsentation der erzielten Einigung stellte der litauische Finanzminister Rimantas Šadžius fest: „,[M]it der Vereinbarung über den einheitlichen Mechanismus zur Bankenabwicklung,

19 Interview im litauischen Finanzministerium, Vilnius, 21. Februar 2014.

20 Die Vereinbarung vom Dezember 2013 sieht eine zehnjährige Übergangszeit vor, bis der Abwicklungsfonds das angestrebte Kapital in Höhe von einem Prozent der gedeckten Einlagen in den teilnehmenden Mitgliedstaaten erreicht hat.

21 Vorschlag für Richtlinie des Europäischen Parlaments und des Rates zur Festlegung eines Rahmens für die Sanierung und Abwicklung von Kreditinstituten und Wertpapierfirmen und zur Änderung der Richtlinien 77/91/ EWG und 82/891/EG des Rates, der Richtlinien 2001/24/EG, 2002/47/EG, 2004/25/EG, 2005/56/EG, 2007/36/ EG und 2011/35/EG sowie der Verordnung (EU) Nr. 1093/2010, KOM (2012) 280 final/2.

22 Europäische Kommission: Vorschlag für eine Richtlinie.../.../ EU des Europäischen Parlaments und des Rates über Einlagensicherungssysteme [Neufassung], KOM (2010) 368. 
ist der Aufbau der Bankenunion fast abgeschlossen. Das ist wahrlich der Höhepunkt der litauischen Präsidentschaft".23

Obwohl sich das Europäische Parlament unzufrieden mit der Einigung über den einheitlichen Mechanismus zur Bankenabwicklung im Rat zeigte, wurde am 20. März 2014, nach 16-stündigen Verhandlungen, ein Kompromiss zwischen Rat und Parlament erzielt. Die Position Deutschlands, dass der gemeinsame Rettungsfonds weder von staatlichen Garantien profitieren noch Zugang zum Europäischen Stabilitätsmechanismus erhalten sollte, setzte sich durch. Ebenso hat die Vereinbarung über den Abwicklungsfonds eine zwischenstaatliche Rechtsnatur erhalten. Die EU-weite Einlagensicherung wurde aus dem Kompromiss herausgenommen, nur die Harmonisierung der nationalen Einlagensicherungsregeln wurde, wie im Dezember 2013 vereinbart, beschlossen. Die Konzessionen an das Europäische Parlament beinhalteten eine Beschleunigung der Einrichtung des Abwicklungsfonds mit einer Verkürzung der Überganszeit von zehn auf acht Jahre, wobei die nationalen Fonds schneller als ursprünglich vorgesehen zusammengeführt werden. Die Zugeständnisse umfassten auch einen Hinweis auf die erweiterte Fähigkeit des Abwicklungsfonds, Kredite am freien Markt aufzunehmen.

Neben der Vermittlung und Aushandlung der Kompromisse zum einheitlichen Abwicklungsmechanismus für Banken, verantwortete die litauische Präsidentschaft auch die Beschlussfassung über die länderspezifischen Empfehlungen im Rahmen des Europäischen Semesters für das Jahr 2014. Ferner begleitete die Präsidentschaft die Erstellung des Berichts über die aus dem Europäischen Semester 2013 gewonnenen Erfahrungen. ${ }^{24}$ Schließlich wurden Gesetzgebungsverfahren in den Bereichen Datenschutz, Europäische Ermittlungsanordnung in Strafsachen, ${ }^{25}$ der Schutz des Euro vor Fälschung ${ }^{26}$ und die Reform der Anforderungen an und Abschlussprüfungen von Jahresabschlüssen von Unternehmen ${ }^{27}$ weiter vorangetrieben.

\section{Der mehrjährige Finanzrahmen 2014-2020: ein wachsendes Europa?}

Die Einigung über den MFR mit dem Europäischen Parlament war eine andere große Aufgabe für den litauischen Vorsitz. Bereits am 3. Juli 2013 beschloss das Europäische Parlament nur über den MFR abzustimmen, wenn eine Einigung über die Anpassung des Haushalts für 2013 und über den Budgetentwurf für 2014 gefunden wird. Als weitere Bedingungen für eine Abstimmung erwartete es ausreichende Fortschritte bei der Einsetzung einer hochrangigen Arbeitsgruppe zur Reform des Eigenmittelsystems sowie bei der Vorbereitung von Durchführungsrechtsakten zum MFR, insbesondere in den Bereichen Landwirtschaft und

23 Litauische Ratspräsidentschaft: Lithuanian Presidency Reaches agreement on Single Resolution Mechanism, Pressemitteilung, 19.12.2013, abrufbar unter: http://www.eu2013.1t/en/news/pressreleases/lithuanian-presiden cy-reaches-agreement-on-single-resolution-mechanism (letzter Zugriff: 6.5.2014).

24 Rat der Europäischen Union: Europäisches Semester - Aus dem Europäischen Semester 2013 gewonnene Erfahrungen und Ausblick, Dok. 14618/13.

25 Das Europäische Parlament/Der Rat: Richtlinie des Europäischen Parlaments und des Rates über die Europäische Ermittlungsanordnung in Strafsachen, PE-CONS 122/13.

26 Europäische Kommission: Vorschlag für eine Richtlinie des Europäischen Parlaments und des Rates zum strafrechtlichen Schutz des Euro und anderer Währungen gegen Geldfälschung und zur Ersetzung des Rahmenbeschlusses 2000/383/JI des Rates, KOM (2013) 42.

27 Europäische Kommission: Vorschlag für Verordnung des Europäischen Parlaments und des Rates über spezifische Anforderungen an die Abschlussprüfung bei Unternehmen von öffentlichem Interesse, KOM (2011) 779; Europäische Kommission: Vorschlag für eine Richtlinie des Europäischen Parlaments und des Rates zur Änderung der Richtlinie 2006/43/EG über Abschlussprüfungen von Jahresabschlüssen und konsolidierten Abschlüssen, KOM (2011) 778. 
Kohäsionspolitik. Die litauische Regierung konzentrierte sich zuerst auf eine Einigung über die Anpassung des Haushalts 2013, die Ende Oktober 2013 erreicht wurde. Parallel arbeitete sie an den Rechtsakten zur Umsetzung des MFR, insbesondere an den Instrumenten für die Kohäsions- und Agrarpolitik, und rief die hochrangige Arbeitsgruppe zu den EU-Eigenmitteln ins Leben, ${ }^{28}$ um einen Kompromiss über den EU-Haushalt 2014 zu erzielen.

Die Einigung über die Mehrheit der Programme und Rechtsakte zur Umsetzung des neuen MFR (über 60 Rechtsakte) wurde im November 2013 erreicht und das Europäische Parlament stimmte zu. Wie die Pressemitteilung des Europäischen Parlaments erklärte, wurden „,[a]lle Bedingungen, die in der Entschließung des Parlaments vom Juli [...] aufgeführt waren, [...] erfüllt“" 29 . Konkret werden die Zustimmung der Mitgliedstaaten zu zusätzlichen 3,9 Milliarden Euro für das Budget 2013, die Einigung über die Rechtsgrundlagen für alle EU-Programme, die auf der Basis der Zustimmung von Rat und dem Parlament beschlossen werden, die Einrichtung der hochrangigen Arbeitsgruppe zur Reform des Eigenmittelsystems, die Vereinbarungen über den flexiblen Einsatz von ungenutzten Geldmitteln in anderen Jahren und über die Flexibilität, Verpflichtungen zwischen den Jahren und Ausgabenkategorien zu bewegen, sowie die Einführung einer Halbzeitüberprüfung des MFR genannt, die die Europäische Kommission verpflichtet, die Funktionsfähigkeit des MFR im Jahr 2016 zu überprüfen. ${ }^{30}$ Die Vereinbarungen über die wichtigsten Programme enthalten Übergangsbestimmungen für die Reform der Gemeinsamen Agrarpolitik im Jahr 2014, eine reformierte Kohäsionspolitik, die über zwei Jahre ausgehandelt wurde, Bestimmungen für die Finanzinstrumente in der EU-Außenpolitik, für die Zusammenarbeit beim Katastrophenschutz, für den Fonds für innere Sicherheit und den Fonds für Asyl und Migration sowie weitere Bestimmungen. Obwohl es zweieinhalb Jahre dauerte, bis die Verhandlungen über den MFR abgeschlossen wurden, wurde der EU-Haushalt für das Jahr 2014 schneller als üblich verabschiedet.

Während der Verhandlungen mit dem Europäischen Parlament über den MFR gab es Fälle, die die Komplexität des Umgangs mit dem Europäischen Parlament illustrieren. Dies hatte sich bereits bei den Verhandlungen der irischen Präsidentschaft gezeigt, als eine Einigung mit dem Verhandlungsführer des Europäischen Parlaments nicht bedeutete, dass der Kompromiss auch von den anderen Abgeordneten unterstützt wurde. Wie der stellvertretende litauische Außenminister Vytautas Leškevičius am 19. November 2013 in Straßburg erklärte: ,the negotiations of unprecedented intensity and difficulty with the EP were one of the biggest challenges of the Lithuanian and previous Presidencies“".31

Weitere Themen, die unter dem Titel ,wachsendes Europa' zusammengefasst wurden, erregten viel weniger öffentliche Aufmerksamkeit. Die Arbeiten an bestimmten Gesetzesvorschlägen machten Fortschritte, wie die gemeinsamen Regeln für Saisonarbeiter, die überarbeitete Tabakrichtlinie, das Abkommen mit dem Europäischen Parlament $\mathrm{zu} \mathrm{CO}_{2}$-Emissionen von PKWs und über das EU-Emissionshandelssystem, die Regulierung von fluorierten Treibhausgasen, die Umsetzung der Digitalen Agenda und auch eine Einigung über den Mehrwert von makroregionalen Strategien. Obwohl keine großen Gesetzesinitiativen im

28 Deren Einsetzung war für Ende 2013 vorgesehen, verzögerte sich aber aufgrund von Uneinigkeit über die Frage, wer der Gruppe vorsitzen soll.

29 Europäisches Parlament: Parlament billigt langfristigen EU-Haushalt für die Jahre 2014-2020, Pressemitteilung, 19.11.2013.

30 Ebenda.

31 Rat der Europäischen Union: Lithuanian Presidency welcomes EP consent to EU multiannual financial Framework, Pressemitteilung, 19.11.2013. 
Bereich der Energiepolitik angestoßen wurden, war es der Präsidentschaft wichtig, dass der Rat zwei Berichte zum Energiebinnenmarkt ${ }^{32}$ und zur Energieaußenpolitik der Europäischen Union $^{33}$ billigte. Genauso befasste sich der Rat der Europäischen Union im Oktober 2013 mit dem Binnenmarkt für Dienstleistungen und der Anwendung des Prinzips der gegenseitigen Anerkennung. Allerdings erhielt die Arbeit an solchen Dossiers im Bereich des Binnenmarkts, wie beispielsweise an der Richtlinie über Zahlungsdienste im Binnenmarkt, ${ }^{34}$ weniger Aufmerksamkeit, da der Fokus auf der Schaffung einer Bankenunion lag.

\section{Die Beziehungen zu den Ländern der Östlichen Partnerschaft: ein offenes Europa}

Die Beziehungen zu den östlichen Partnerländern standen während der litauischen Präsidentschaft außergewöhnlich hoch auf der Agenda der Europäischen Union. Dies war zum Teil eine Folge der Einbindung Litauens in die Östliche Partnerschaft, aber auch dem politischen Kalender und Ereignissen in der östlichen Nachbarschaft selbst geschuldet. Im Gegensatz zum MFR und zur Bankenunion betrachtete die litauische Regierung die Östliche Partnerschaft als das ,Leuchtturmprojekt ${ }^{`}$ ihrer Ratspräsidentschaft und die meisten Medien berichteten über dieses Thema während der Präsidentschaft.

Eine Reihe der litauischen Ziele und Erwartungen bezüglich der Östlichen Partnerschaft wurden realisiert. Assoziierungsabkommen, einschließlich vertiefter und umfassender Freihandelszonen, wurden mit der Republik Moldau und Georgien paraphiert und zum ersten Mal in den vier Jahren seit Gründung der Östlichen Partnerschaft waren alle Mitgliedsländer beim Gipfel der Östlichen Partnerschaft vertreten. Die Einladungen für den Gipfel waren an Staaten adressiert und nicht an spezifische Personen, um dem Vorwurf der ,Diskriminierung ‘ Weißrusslands vorzubeugen, was der Fall während des Gipfels im Jahr 2011 gewesen war. Die gemeinsame Erklärung, ${ }^{35}$ die während des Gipfels angenommen wurde, fasst die Leistungen der Politik der Östlichen Partnerschaft zusammen und nennt mehrere Schritte, um die Beziehungen der Europäischen Union zu den Staaten ihrer östlichen Nachbarschaft bis zum nächsten Gipfel im Jahr 2015 zu vertiefen. Darunter sind weitere Reformen des Justizwesens und die Stärkung der Strafverfolgung, die Unterzeichnung von Assoziierungsabkommen bis Herbst 2014, das Einsetzen einer Assoziierungsagenda und die Fortentwicklung der Zusammenarbeit in einer Reihe von Politikbereichen, wie der Visa-Liberalisierung, Wirtschaft, Wissenschaft und Innovation, Landwirtschaft, Umwelt, Verkehr, Verteidigung und Energie. Die Teilnehmer waren sich einig, dass die Europäische Union dem Anreiz basierten Ansatz folgend weiterhin die Reformen in den Partnerländern durch Finanzhilfen unterstützt, unter Berücksichtigung der vorläufigen Anwendung und Umsetzung der Assoziierungsabkommen. Dabei war die Hochrangigkeit der Vertreter während des Vilnius-Gipfels mit 34 Staats- und Regierungschefs sowie Ministern bemerkenswert.

32 Europäische Kommission: Mitteilung der Kommission an das Europäische Parlament, den Rat, den Europäischen Wirtschafts- und Sozialausschuss und den Ausschuss der Regionen. Ein funktionierender Energiebinnenmarkt, KOM (2012) 663.

33 Europäische Kommission: Bericht der Kommission an das Europäische Parlament, den Rat und den Europäischen Wirtschafts- und Sozialausschuss. Umsetzung der Mitteilung zur Energieversorgungssicherheit und internationalen Zusammenarbeit sowie der Schlussfolgerungen des Rates „Energie“ vom November 2011, KOM (2013) 638.

34 Europäische Kommission: Vorschlag für eine Richtlinie des Europäischen Parlaments und des Rates über Zahlungsdienste im Binnenmarkt, zur Änderung der Richtlinien 2002/65/EG, 2013/36/EU und 2009/110/EG sowie zur Aufhebung der Richtlinie 2007/64/EG, KOM (2013) 547.

35 Rat der Europäischen Union: Joint Declaration of the Eastern Partnership Summit, Vilnius, 28-29 November 2013. Eastern Partnership: the way ahead, Dok. 17130/13. 
Die genaue Formulierung, wie die östlichen Partnerländer bezeichnet wurden, war wie immer ein heikles Thema. Litauen und einige andere EU-Mitgliedstaaten wollten ihnen eine mehr oder weniger explizite Mitgliedschaftsperspektive geben. ${ }^{36}$ Dies war nicht realisierbar, aber eine vorsichtige Entwicklung in diese Richtung ist dennoch zu beobachten: Im Gegensatz zu den gemeinsamen Erklärungen von 2009 und 2011 wurden die östlichen Partnerländer nicht mehr als „osteuropäische Partner“ oder „Partnerländer“, sondern als „osteuropäische Länder, Teilnehmerstaaten der Östlichen Partnerschaft“ bezeichnet. Die östlichen Partnerländer wurden damit immer noch nicht als ,europäische Staaten' bezeichnet, was zu sehr der Formulierung des Art. 49 Vertrag über die Europäische Union ähneln würde, aber das Wort ,Staat‘ wurde unmittelbar nach der Formulierung ,osteuropäische Länder' erwähnt.

Fortschritte wurden auch hinsichtlich der Mobilität der Menschen zwischen der Europäischen Union und den Ländern der Östlichen Partnerschaft erzielt: Verhandlungen über Visaerleichterungen und Rückübernahmeabkommen mit Aserbaidschan wurden abgeschlossen und ein ähnliches Abkommen mit Armenien wurde im Europäischen Parlament angenommen. In der letzten Sitzung des Ausschusses der Ständigen Vertreter während der litauischen Präsidentschaft gelang es, eine politische Einigung über die Aufhebung der Visumspflicht für die Republik Moldau zu erreichen. ${ }^{37}$ Zur Überraschung vieler erklärten auch die Vertreter Weißrusslands während des Gipfels zur Östlichen Partnerschaft, dass sie bereit wären, Verhandlungen über Visaerleichterungen und über ein Rückübernahmeabkommen zu beginnen.

Die Präsidentschaft hat weitgehend zur Ausweitung der sektoralen Zusammenarbeit im Rahmen der Östlichen Partnerschaft beigetragen. Die litauische Regierung organisierte mehrere Veranstaltungen zum Thema Verteidigungszusammenarbeit mit den Ländern der Östlichen Partnerschaft. Konkrete Erfolge waren die Einrichtung des Panels zur Gemeinsamen Sicherheits- und Verteidigungspolitik im Rahmen der multilateralen Plattform I („Demokratie, Good Governance und Stabilität") sowie die Unterzeichnung des Rahmenabkommens über die Beteiligung der Republik Moldau und Georgiens an der Gemeinsamen Sicherheitsund Verteidigungspolitik. Im September 2013 veranstaltete die Ratspräsidentschaft eine Konferenz zur Stärkung der Zusammenarbeit der Europäischen Union mit den Ländern der Östlichen Partnerschaft in der Forschungspolitik mit dem Ergebnis, ein Panel für Forschung und Innovation im Rahmen der multilateralen Plattform IV („Kontakte zwischen den Menschen") zu schaffen, wo unter anderem die mögliche Assoziierung der Länder der Östlichen Partnerschaft mit dem aktuellen Rahmenprogramm für Forschung und Innovation „Horizont 2020“ der Europäischen Union diskutiert wird.

Die litauische Präsidentschaft unternahm auch erhebliche Anstrengungen, um die $\mathrm{Zu}-$ sammenarbeit im Bereich Justiz und Inneres im Rahmen der Östlichen Partnerschaft zu stärken. ,Vilnius“ war Gastgeber der jährlichen Präsidentschaftskonferenz der Europäischen Polizeiakademie (EPA) im September 2013, zu der die östlichen Partnerländer eingeladen wurden. ${ }^{38}$ Die Präsidentschaft organisierte ein Treffen der Justiz- und Innenminister der Europäischen Union und der Länder der Östlichen Partnerschaft, das erste seiner Art. Dessen

36 Radio Free Europe/Radio Liberty: Draft EU Summit Text Acknowledges ,Aspirations‘ Of Eastern Neighbors, abrufbar unter: http://www.rferl.org/content/eu-neighbors-eastern-statement/25153908.html (letzter Zugriff: 6.5.2014).

37 Litauische Ratspräsidentschaft: Lithuanian Presidency reaches agreement on visa free travels for the Republic of Moldova, abrufbar unter: http://www.eu2013.1t/en//news/pressreleases/lithuanian-presidency-reaches-agreement-on-visa-free-travels-for-the-republic-of-moldova (letzter Zugriff: 20.12.2013).

38 European Police College: The international dimension of law enforcement cooperation: The Lithuanian Presidency conference, abrufbar unter: www.cepol.europa.eu/index.php?id=news-details\&tx_ttnews\%5Btt_news $\% 5 \mathrm{D}=543 \& \mathrm{cHash}=351718 \mathrm{fafc} 31018 \mathrm{~d} 2693011$ fedfbbb09 (letzter Zugriff: 20.2.2014). 
Teilnehmer billigten eine gemeinsame Erklärung, ${ }^{39}$ in der eine Bilanz der bisherigen Fortschritte in den jeweiligen Politikbereichen gezogen und die Bereiche für zukünftige gemeinsame Anstrengungen skizziert werden, einschließlich der justiziellen Zusammenarbeit in Zivil- und Strafsachen sowie der Zusammenarbeit in Fragen von organisierter und grenzüberschreitender Kriminalität, Korruption, Drogenkriminalität, Datenschutz, Cyber-Kriminalität, Migration und Mobilität. Während der Sitzung kündigte Georgien an, ein Folgetreffen im Jahr $2014 \mathrm{zu}$ organisieren; ${ }^{40}$ in Abhängigkeit von der politischen Situation in den Ländern der Östlichen Partnerschaft, ist die Fortsetzung dieses hochrangigen Dialogs über Justiz und Inneres auf einer dauerhafteren Grundlage wahrscheinlich.

Schließlich investierte die litauische Regierung viel in einen verstärkten Dialog mit den Ländern der Östlichen Partnerschaft zum Thema Verkehrspolitik. Der Höhepunkt in diesem Bereich war das Treffen der Verkehrsminister der Europäischen Union und der Länder der Östlichen Partnerschaft im Oktober 2013, bei dem die Teilnehmer eine gemeinsame Erklärung annahmen ${ }^{41}$ und dem Entwurf eines regionalen Transportnetzwerks der Östlichen Partnerschaft zustimmten, einschließlich einer Liste vorrangiger Straßen- und Schienengrenzübergänge, Häfen und Flughäfen. Die gemeinsame Erklärung verlangt de facto auch eine Ausweitung der Gemeinsamen Leitlinien für den Aufbau eines transeuropäischen Verkehrsnetzes auf die Länder der Östlichen Partnerschaft. Eine weitere Errungenschaft in der Transportzusammenarbeit war die Paraphierung eines EU-Ukraine-Luftverkehrsabkommens während des Gipfels im Rahmen der Östlichen Partnerschaft, welches gemeinsame Standards für die Fluggesellschaften festlegt, was den Weg für die Schaffung eines gemeinsamen Luftverkehrsraums freimacht.

Neben diesen Erfolgen sind während der litauischen Präsidentschaft auch zwei Misserfolge in der Politik gegenüber der Östlichen Partnerschaft zu verzeichnen: Ein erster Rückschlag war die Entscheidung des armenischen Präsidenten Serzh Sargsyan im September 2013, der gemeinsamen Zollunion von Russland, Weißrussland und Kasachstan beizutreten; damit verbunden war die Ablehnung der vorgesehenen Paraphierung eines Assoziierungsabkommens mit der Europäischen Union. Der zweite Misserfolg bestand in der Weigerung des ukrainischen Präsidenten Victor Janukowitsch, das Assoziierungsabkommen während des Gipfels der Östlichen Partnerschaft zu unterzeichnen. Beide Entscheidungen waren große Enttäuschungen für die Europäische Union im Allgemeinen und die litauische Präsidentschaft im Besonderen. Die Entscheidung von Janukowitsch löste eine Reihe von Ereignissen aus, ${ }^{42}$ die von EU-Entscheidungsträgern definitiv nicht erwartet wurden. Bis zu einem gewissen Grad veranschaulicht dies, dass die Politik der Östlichen Partnerschaft die geopolitische Komplexität der Region unterschätzt und das Verhalten von Präsident Janukowitsch falsch interpretiert hatte. Wie sich herausstellte, meinte er es mit dem Assoziierungsabkommen nicht ernst, sondern spielte vielmehr ein innenpolitisches Spiel und versuchte, so viel finanzielle Unterstützung wie möglich von der Europäischen Union und Russland zu erhalten.

39 Rat der Europäischen Union: Joint Declaration on Eastern Partnership Justice and Home Affairs, Dok. 14558/13.

40 Interview im litauischen Justizministerium, Vilnius, 18. Februar 2014.

41 Litauisches Verkehrsministerium: Joint Declaration - Future of Eastern Partnership transport cooperation, abrufbar unter: http://www.sumin.lt/files/uploads//Rytu\%20partnerystes\%20deklaracija.pdf (letzter Zugriff: 10.10.2013).

42 Für eine detaillierte Analyse der Folgen dieser Entscheidung siehe Katrin Böttger: Auf dem sicherheitspolitischen Auge blind: Die EU-Außenpolitik angesichts der Ukraine-Krise: Zustand und Entwicklungsoptionen, in diesem Band, S. 95-108. 
Ein weiteres Ereignis, das weniger mediale Aufmerksamkeit erhielt und bei dem die Europäische Union eine zweifelhafte Rolle während der litauischen Präsidentschaft spielte, war die Parlamentswahl in Aserbaidschan im Oktober 2013. In einem Skandal mit dem Spitznamen ,Appgate' wurde das ,Ergebnis' der Wahl über ein entsprechendes Softwareprogramm versehentlich bereits einen Tag vor den Wahlen verkündet. ${ }^{43}$ Die internationalen Reaktionen waren gemischt: Das Europäische Parlament und die Parlamentarische Versammlung des Europarates nannte die Wahlen „frei, fair und transparent" ${ }^{44}$ während das OSZE-Büro ${ }^{45}$ für demokratische Institutionen und Menschenrechte und die Parlamentarische Versammlung der OSZE feststellten, dass die „Wahl von Beschränkungen der Meinungs-, Versammlungs- und Vereinigungsfreiheit untergraben wurde, so dass gleiche Wettbewerbsbedingungen für Kandidaten nicht garantiert waren“. ${ }^{46}$ Die Sprecher der Hohen Vertreterin der Union für Außen- und Sicherheitspolitik Catherine Ashton und des EUKommissars für Erweiterung und Europäische Nachbarschaftspolitik Štefan Füle lobten die hohe Wahlbeteiligung, verwiesen aber auch auf die Probleme, die von der OSZE und deren Beobachtern kritisiert wurden. ${ }^{47}$ Bemerkenswerterweise hielt sich die Ratspräsidentschaft in Bezug auf die Wahl bedeckt; eine mögliche Erklärung dafür könnte sein, dass ein ,AntiAzeri'-Standpunkt leicht als ,pro-armenische' Sicht interpretiert werden kann und Litauen immer versucht, den Eindruck zu vermeiden, es unterstütze eine der Seiten in diesem bilateralen Konflikt. ${ }^{48}$

\section{Litauens Rollen während der Präsidentschaft}

\section{Sechs mögliche Rollen einer Ratspräsidentschaft}

Die einzige formale Verantwortung der Präsidentschaft besteht in der Organisation und Leitung der Sitzungen des Rates der Europäischen Union und seiner Vorbereitungsgremien. Allerdings wurde die rotierende Präsidentschaft nach und nach ein wichtiger Akteur im EUEntscheidungsprozess, wobei sie dabei sechs mögliche Rollen wahrnimmt. ${ }^{49}$ Die erste offizielle Rolle ist die des Veranstalters beziehungsweise Managers. Zweitens gestaltet das jeweils amtierende Land die Tagesordnung des Rates. Da die Ressourcen begrenzt sind, müssen Prioritäten gesetzt werden - eine Aufgabe, die die Präsidentschaft gemeinsam mit der Europäischen Kommission wahrnimmt. Die Präsidentschaft betont bestimmte Themen und nimmt manchmal andere Themen von der Tagesordnung. Drittens erfüllt die Präsidentschaft die Rolle eines Vermittlers, der einerseits Konsens zwischen den Mitgliedstaaten innerhalb des Rates herstellt und andererseits zwischen dem Rat und den anderen EU-Organen vermittelt. Viertens hat die Präsidentschaft eine Rolle als Repräsentant: Sie spricht im Namen

43 Andrew Rettman: EU ponders reaction to Azerbaijan's ,appgate', in: EUObserver, 10.10.2013.

44 Europarat: Presidential election in Azerbaijan: joint statement by PACE and EP delegations, 2013.

45 Organisation für Sicherheit und Zusammenarbeit in Europa.

46 Organisation für Sicherheit und Zusammenarbeit in Europa: International Election Observation Mission, Republic of Azerbaijan, Presidential Election, 9 October 2013: Statement of Preliminary Findings and Conclusions.

47 Europäische Kommission: Statement by the Spokespersons of EU High Representative Catherine Ashton and Commissioner Štefan Füle on Presidential elections in Azerbaijan, MEMO/13/879.

48 Interview im litauischen Außenministerium, Vilnius, 21. Februar 2014.

49 Bo Bjurulf: How did Sweden Manage the European Union?, Zentrum für Europäische Integrationsforschung: ZEI Discussion Papers C 96/2001; Adriaan Schout/Sophie Vanhoonacker: Evaluating Presidencies of the Council of the EU: Revisiting Nice, in: Journal of Common Market Studies 5/2006, S. 1051-1077; Ole Elgström: Introduction, in: Ole Elgström (Hrsg.): European Union Council Presidencies: A comparative perspective, London 2003, S. 1-17; Ole Elgström: The Presidency: The Role(s) of the Chair in European Union Negotiations, in: The Hague Journal of Diplomacy 1/2006, S. 171-195. 
des Rates und vertritt die Europäische Union in den Medien. Vor dem Inkrafttreten des Vertrags von Lissabon im Jahr 2009 vertrat die Präsidentschaft die Europäische Union auch gegenüber Drittstaaten und internationalen Organisationen. Seitdem wird diese Rolle auf Ministerebene meist von Ashton, der Hohen Vertreterin der Union für Außen- und Sicherheitspolitik und Vizepräsidentin der Europäischen Kommission, und auf der Ebene der Staats- und Regierungschefs von Herman Van Rompuy, dem ständigen Präsidenten des Europäischen Rates, und José Manuel Barroso, dem Präsidenten der Europäischen Kommission, ausgeübt. Fünftens, wenn auch allgemein weniger anerkannt, wird von der Präsidentschaft auch politische Führung erwartet, das heißt, dass sie die weitere Integration und/oder ein besseres Funktionieren der Europäischen Union befördert. Schließlich handelt die Präsidentschaft manchmal als ein Vertreter nationaler Interessen. Da das amtierende Land einen privilegierten Zugang zu Informationen und zum Verhandlungsprozess hat, können die Entscheidungsträger versucht sein, Entscheidungen nach ihren eigenen Vorlieben zu beeinflussen. Diese Rolle ist die umstrittenste, da erwartet wird, dass der Vorsitz neutral ist, nicht nur entsprechend dem Präsidentschaftshandbuch des Ratssekretariats, ${ }^{50}$ sondern auch nach informell anerkannten Normen im Rat.

\section{Litauen: erfolgreicher Verwalter, Vermittler in Finanzfragen, Agenda-Setter und politischer Führer in der Östlichen Partnerschaft}

Die wahrscheinlich arbeitsintensivste, aber auch am wenigsten sichtbarste Rolle der Präsidentschaft ist die als Manager. In der Tat wird diese Rolle nur sichtbar, wenn etwas schief geht: „while failures are often attributed to particular presidencies, successes are not: they always have several mothers and fathers. " 51 In diesem Sinne kann die litauische Präsidentschaft als Erfolg bezeichnet werden: Es gab keine Probleme mit dem administrativen Teil der Präsidentschaft. Zusätzlich zu dieser sehr wichtigen Rolle als Manager, hingen die Rollen, die der litauische Vorsitz wahrzunehmen hatte, von bestimmten Dossiers ab.

In den Verhandlungen über den MFR und die Bankenunion trat die litauische Präsidentschaft aktiv als Vermittler auf und versuchte, eine Einigung zwischen dem Rat und dem Europäischen Parlament (im Fall des MFR) und zwischen den Mitgliedstaaten innerhalb des Rates (im Fall der Bankenunion) zu erzielen. Sie erfüllte die Erwartungen, die in diesem Bereich an sie gestellt wurden, vollkommen. Dabei ist zu bedenken, dass das Europäische Parlament, zunehmend selbstbewusst agiert und versucht, seine durch den Vertrag von Lissabon erweiterte institutionelle Macht zu maximieren. Außerdem demonstrierte die litauische Präsidentschaft bei bestimmten Themen, wie dem einheitlichen Mechanismus zur Bankenabwicklung oder der Frage der $\mathrm{CO}_{2}$-Emissionen, Führung und Entschlossenheit um umfassende Kompromisse zu erzielen.

In der Politik der Östlichen Partnerschaft konzentrierte sich die litauische Präsidentschaft auf die Rollen des Agenda-Setters und politischen Führers. Obwohl die Prioritäten im Bereich der Östlichen Partnerschaft weitgehend den nationalen Interessen Litauens entsprachen, verfolgten die litauischen Entscheidungsträger diese konsequent als europäische und nicht als nationale Prioritäten. Litauen konnte mit Erfolg langfristig angelegte Punkte auf die Agenda der Politik der Östlichen Partnerschaft setzen. Durch die Priorisierung der Region und die Organisation zahlreicher Veranstaltungen auf verschiedenen Ebenen gelang es der

50 Rat der Europäischen Union: Council Guide, I. The Presidency Handbook, 2006.

51 Hanna Ojanen/Eero Vuohula: The Finnish Presidency: Efficiency Before Eloquence?, in: Journal of Common Market Studies, Annual Review 2007, S. 17-24. 
Präsidentschaft, die Aufmerksamkeit aller EU-Staaten auf die Länder der Östlichen Partnerschaft zu lenken. Mehrere neue formelle und informelle Foren für die Zusammenarbeit wurden eingerichtet. Die Anwesenheit von Ministern, stellvertretenden Ministern und Experten bei diesen Treffen sorgte dafür, dass die Initiativen erhebliche politische Unterstützung erhielten, die bei der weiteren Entwicklung der Zusammenarbeit der Europäischen Union mit ihren östlichen Nachbarn helfen wird. Zusammenfassend kann somit festgestellt werden, dass Litauen mit der eigens akzentuierten außenpolitischen Agenda der Europäischen Union eine politische Führungsrolle zur Förderung europäischer Zusammenarbeit und Integration im weitesten Sinne einnahm.

Was die mögliche Durchsetzung litauischer Interessen während des Vorsitzes betrifft, ist festzustellen, dass die litauischen Entscheidungsträger nicht versuchten, ihre nationale Agenda durchzusetzen, und so die ,Neutralitätsnorm` einhielten. Es gibt keine Dossiers, in denen die Präsidentschaft klar versuchte, nationale Interessen durchzusetzen. Auch wenn Fragen von nationaler Bedeutung hervorgehoben wurden, wie die Östliche Partnerschaft, wurde dies in einer Weise getan, die ihre Bedeutung für die gesamte Europäische Union zeigte. Die Tatsache, dass wichtige Ereignisse, wie Ministertreffen, in Brüssel und nicht in Vilnius stattfanden, zeigt, dass Litauen diese als ,europäische“ und nicht als ,nationale' Themen fördern wollte.

\section{Schlussfolgerungen: die Herausforderung, die gewonnenen Vorteile zu erhalten}

Litauens Ratspräsidentschaft fand unter Rahmenbedingungen statt, die ihre Spielräume einschränkte. Von besonderer Bedeutung waren die legislativen und politischen Kalender der Europäischen Union, die die Arbeitsbelastung für die litauische Regierung deutlich erhöhten. Trotz dieses schwierigen Umfelds gelang es der Präsidentschaft, alle geplanten wichtigen Dossiers abzuschließen.

Es ist klar, dass die Präsidentschaft in verschiedenen Dossiers unterschiedliche Rollen ausübte: die Rolle des Vermittlers und Schlichters bei den Verhandlungen über den MFR und die Bankenunion sowie die Rolle des Agenda-Setters und politischen Führers in der Außenpolitik, vor allem in der Östlichen Partnerschaft. Die von der Präsidentschaft gespielte Rolle hängt nicht (nur) von den nationalen Interessen des jeweils amtierenden Landes ab, sondern auch von den Merkmalen der Dossiers, den Entscheidungsprozessen und den beteiligten Interessengruppen. Einige Dossiers - wie die Politik der Östlichen Partnerschaft sind stärker abhängig vom politischen Beitrag bestimmter Mitgliedstaaten und der Präsidentschaft, während andere Dossiers - wie die Verhandlungen über den Haushalt - einerseits eine intensive Vermittlung zwischen den Mitgliedstaaten und andererseits zwischen dem Rat und anderen EU-Organen erfordern. Hier erwies sich die litauische Präsidentschaft als eine ,normale ' Präsidentschaft, das heißt eine effiziente Präsidentschaft, die sich wandelnden Gegebenheiten und Anforderungen anpasste und die erwarteten Aufgaben erfüllte.

Während die Präsidentschaft der litauischen Regierung eine Gelegenheit bot, während ihrer Amtszeit in der Europäischen Union sichtbarer und einflussreicher zu werden, hat diese Erfahrung auch mögliche langfristige Auswirkungen auf die nationale Verwaltung und Litauens Rolle in der Europäischen Union. Die langfristigen Vorteile der Durchführung einer Präsidentschaft sind in der Literatur gut dokumentiert. ${ }^{52}$ Eine große Zahl litauischer Beamter profitierte von den direkten Erfahrungen mit den EU-Entscheidungsprozessen. Die Ratsprä-

52 Simone Bunse: Small States and EU Governance: Leadership through the Council Presidency, New York 2009. 
sidentschaft hat zu einem besseren Verständnis und Bewusstsein für die EU-Politik geführt, eine aktivere Beteiligung der litauischen Regierung an EU-Dossiers und eine deutliche Erweiterung der Netzwerke des Landes mit anderen Mitgliedstaaten und den EU-Institutionen ermöglicht. Dies gilt für die Beamten in der Ständigen Vertretung Litauens bei der Europäischen Union und in Vilnius, die mit bestimmten Dossiers der Präsidentschaft befasst waren. Zusätzlich betrieb die litauische Ministerialbürokratie auch aktiv eine Erweiterung von Netzwerken und den Aufbau von Expertise indem einige Beamte während der Vorbereitung auf die Präsidentschaft nach Irland entsandt wurden, andere wurden für eine Mitarbeit in wichtigen Institutionen der Europäischen Union, wie der Europäischen Kommission und dem Europäischen Auswärtigen Dienst, zeitweise abgestellt. ${ }^{53}$ Eine zentrale Herausforderung wird es nun sein, diese Netzwerke und Expertise zu erhalten und damit die langfristigen positiven Auswirkungen der Präsidentschaft für Litauen zu bestätigen.

Übersetzung aus dem Englischen: Karin Göldner.

53 Interview mit einem EU-Diplomaten, Vilnius, 12. März 2014; Interview im litauischen Verteidigungsministerium, Vilnius, 14. März 2014; Interview im litauischen Wissenschafts- und Bildungsministerium, Vilnius, 18. März 2014. 